\title{
Very Low-Mass Stars with Extremely Low Metallicity in the Milky Way's Halo
}

\author{
Wako Aoki ${ }^{1}$, Timothy C. Beers ${ }^{2}$, Takuma Suda ${ }^{3}$, Satoshi Honda ${ }^{4}$ and \\ Young Sun Lee ${ }^{5}$ \\ ${ }^{1}$ National Astronomical Observatory of Japan, \\ Mitaka, Tokyo, Japan \\ email: aoki.wako@nao.ac.jp \\ ${ }^{2}$ University of Notre Dame, Notre Dame, IN, United States \\ ${ }^{3}$ University of Tokyo, Tokyo, Japan \\ ${ }^{4}$ University of Hyogo, Sayo, Hyogo, Japan \\ ${ }^{5}$ Chungnam National University, Daejeon 305-764, Republic of Korea
}

\begin{abstract}
Large surveys and follow-up spectroscopic studies in the past few decades have been providing chemical abundance data for a growing number of very metal-poor $([\mathrm{Fe} / \mathrm{H}]<$ -2) stars. Most of them are red giants or main-sequence turn-off stars having masses near 0.8 solar masses. Lower mass stars with extremely low metallicity $([\mathrm{Fe} / \mathrm{H}]<-3)$ are yet to be explored. Our high-resolution spectroscopic study for very metal-poor stars found with SDSS has identified four cool main-sequence stars with $[\mathrm{Fe} / \mathrm{H}]<-2.5$ among 137 objects (Aoki et al. 2013). The effective temperatures of these stars are $4500-5000 \mathrm{~K}$, corresponding to a mass of around 0.5 solar masses. Our standard analysis of the high-resolution spectra based on 1D-LTE model atmospheres has obtained self-consistent chemical abundances for these objects, assuming small values of micro-turbulent velocities compared with giants and turn-off stars. The low temperature of the atmospheres of these objects enables us to measure their detailed chemical abundances. Interestingly, two of the four stars have extreme chemical-abundance patterns: one has the largest excesses of heavy neutron-capture elements associated with the $r$-process abundance pattern known to date (Aoki et al. 2010), and the other exhibits low abundances of the $\alpha$-elements and odd- $Z$ elements, suggested to be signatures of the yields of very massive stars (> 100 solar masses; Aoki et al. 2014). Although the sample size is still small, these results indicate the potential of very low-mass stars as probes to study the early stages of the Milky Way's halo formation.
\end{abstract}

Keywords. stars:abundances, stars:fundamental parameters, stars:individual(SDSS J001820.51093939.2, stars:low-mass, stars:population II, stars:subdwarfs)

\section{Introduction}

Very metal-poor stars are believed to be ancient objects that were born in the early phases of the Milky Way's formation. They should be low-mass $\left(M<1 \mathrm{M}_{\odot}\right)$ stars with long lifetimes ( $>10$ billion years), containing rich information about the nucleosynthetic yields of the first generations of stars and early chemical evolution, as well as the assembly history of the Milky Way. The large-scale surveys of very metal-poor stars and followup high resolution spectroscopy in the past few decades have accumulated chemical abundance data for a large number of stars (Frebel \& Norris 2015). According to the SAGA database (Suda et al. 2008), which is collecting information on metal-poor stars, chemical compositions have been reported for about 1000 stars with $[\mathrm{Fe} / \mathrm{H}]<-2.5$. Most of the stars studied so far are red giants or main-sequence turn-off stars. By comparison, 
Table 1. Cool main-sequence, very metal-poor stars found with SDSS and Subaru

\begin{tabular}{llcccccc}
\hline Object & Object Name & $T_{\text {eff }}$ & $\log g$ & {$[\mathbf{F e} / \mathbf{H}]$} & $v_{\text {Helio }}$ & $M$ & $d$ \\
\hline & & $(\mathrm{K})$ & & & $\left(\mathrm{km} \mathrm{s}^{-1}\right)$ & $\left(\mathrm{M}_{\odot}\right)$ & $(\mathrm{pc})$ \\
\hline SDSS J0018-0939 & SDSS J001820.51-093939.2 & 4600 & 5.0 & -2.5 & -122.9 & 0.47 & 300 \\
SDSS J0259+0057 & SDSS J025956.45+005713.3 & 4550 & 5.0 & -3.3 & +35.7 & 0.47 & 380 \\
SDSS J1703+2836 & SDSS J170339.60+283649.9 & 5000 & 4.8 & -3.2 & -147.9 & 0.52 & 370 \\
SDSS J2357-0052 & SDSS J235718.91-005247.8 & 5000 & 4.8 & -3.4 & -9.4 & 0.52 & 440 \\
\hline
\end{tabular}

Notes:

Stellar name, atmospheric parameters, and radial velocities are taken from Aoki et al. (2013). Stellar mass and luminosity are estimated from isochrones (Kim et al. 2002), and the distance is estimated from the luminosity and the apparent magnitude.

studies of cool main-sequence stars with very low metallicity are quite limited (e.g., Yong et al. 2003a).

This is not surprising, given the faintness of the cool main-sequence stars. According to isochrones for very low metallicity $([\mathrm{Fe} / \mathrm{H}]=-3.5$; e.g., Kim et al. 2002$)$, red giants with effective temperature $\left(T_{\text {eff }}\right)$ of $5000 \mathrm{~K}\left(0.8 \mathrm{M}_{\odot}\right)$ have luminosity $(L)$ as high as $100-200 \mathrm{~L}_{\odot}$, and main-sequence stars with $T_{\text {eff }}=6000 \mathrm{~K}$ have $0.7 \mathrm{~L} \odot$. The luminosity of cool main-sequence stars with $T_{\text {eff }}$ of $5000 \mathrm{~K}\left(0.5 \mathrm{M}_{\odot}\right)$ is only $0.1 \mathrm{~L}_{\odot}$. Hence, the small sample of cool main-sequence stars with extremely low metallicity obtained so far does not indicate that very low-mass stars are not formed at low metallicity, just that they are difficult to find. Future surveys of metal-poor stars including cool main-sequence stars have the potential to constrain the initial mass function of low-mass stars as a function of metallicity.

Cool main-sequence stars are also useful to study molecular features that provide unique opportunities to measure elemental and isotopic abundances. For instance, $\mathrm{MgH}$ features have been systematically measured by Yong et al. (2003b) to investigate Mg isotope ratios.

\section{Low-mass very metal-poor stars found with SDSS and Subaru}

We have conducted high-resolution spectroscopy for candidate very metal-poor stars found with Sloan Digital Sky Survey (SDSS; York et al. 2000). The results obtained for the full sample (137 stars) were reported by Aoki et al. (2013). The sample includes four main-sequence stars with $T_{\text {eff }}=4500-5000 \mathrm{~K}$ (Table 1 ).

The surface gravities of these stars are estimated by demanding that the Fe abundances derived from neutral and ionized species (i.e., Fe I and Fe II lines) are consistent. For SDSS J 0018-0939, the value obtained by this assumption is slightly larger than $\log g=$ 5.0. For this star $\log g=5.0$, the value expected from the isochrone, is adopted.

Estimates of $\mathrm{Fe}$ abundances from SDSS spectra are compared with the determination from the high-resolution spectra obtained with Subaru/HDS in Figure 1 of Aoki et al. (2013). The comparison shows no significant offset in general, although the scatter is as large as 0.5 dex for extremely metal-poor stars. The Fe abundances estimated from the SDSS spectra for the four cool main-sequence stars are, however, systematically lower than the values obtained by the analyses of high-resolution spectra. One reason for this discrepancy is the difference in the gravity estimates: the high-resolution spectra provide clear evidence for high gravity $(\log g \sim 5$ ), such as the weakness of ionized Fe features and the broad wings of strong absorption lines. Such information was not available in the pipeline analyses for SDSS data (Lee et al. 2008), and relatively low gravities $(\log g \sim 3-4)$ 
was derived. Further calibration will be useful for the analysis of SDSS spectra of cool main-sequence stars.

\section{A signature of very massive first stars}

Chemical-abundance ratios obtained for three of the four cool main-sequence stars studied by Aoki et al. (2013) are similar to those found for main-sequence turn-off stars and red giants (Fig. 1). Aoki et al. (2014) also confirmed that our analysis for the comparison star G 39-36, which is a bright object with similar stellar parameters, obtains similar results. The abundance patterns of these stars are well-explained by the yields of core-collapse supernovae with mass of several tens of solar masses (Fig. 1).

However, among the four cool main-sequence stars found in our SDSS/Subaru sample, SDSS J 0018-0039 exhibits peculiar chemical abundance patterns (Aoki et al. 2014). While the $\mathrm{Fe}$ abundance of this star is not extreme $([\mathrm{Fe} / \mathrm{H}] \sim-2.5)$, light elements such as $\mathrm{C}$ and $\mathrm{Mg}$ are deficient $([\mathrm{C} / \mathrm{Fe}]=-0.8$ and $[\mathrm{Mg} / \mathrm{Fe}]=-0.5)$. The low abundance of $\mathrm{Co}([\mathrm{Co} / \mathrm{Fe}]=-0.7)$ is also remarkable. This abundance pattern is not explained by nucleosynthesis models for the usual core-collapse supernovae. Instead, Aoki et al. (2014) discussed that an explosion of a very massive star $\left(>100 \mathrm{M}_{\odot}\right)$ is a possible source of this peculiar abundance pattern (Fig. 2).

In order to investigate the abundance pattern in more detail, we obtained a highresolution $(R=60,000)$ spectrum, with higher $\mathrm{S} / \mathrm{N}$ ratio, with Subaru/HDS in August 2014. A preliminary analysis of the new spectrum confirms the abundance properties of this star. New results obtained from the high-S/N spectrum are as follows;

- Fe abundances from ionized species: The Fe abundances obtained from absorption lines of neutral and singly ionized Fe exhibit a discrepancy in the previous analysis if $\log g=5.0$ is adopted. In order to obtain consistent Fe abundances from both species, a higher $\log g$ value needs to be assumed, which is not realistic according to isochrones for metal-poor main-sequence stars (Kim et al. 2002). This problem is not solved by the analysis of the new spectrum. The number of Fe II lines detected is, however, still quite small, and the result is not very conclusive. It should be noted that the Ti abundances derived from Ti I and Ti II show good agreement if $\log g=5.0$ is adopted.

- Si abundance: The Si abundance, estimated from the saturated Si I line at $3906 \AA$ in the previous study (Aoki et al. 2014 ), is quite low ( $[\mathrm{Si} / \mathrm{Fe}]=-0.4)$. The preliminary analysis of another Si line (Si I $4102 \AA$ ) for our new spectrum confirms the underabundance of $\mathrm{Si}$. The result is slightly dependent on the treatment of contamination of the $\mathrm{SiH}$ molecular feature, and more careful analysis is required to obtain a final result.

- $\mathrm{MgH}$ feature: Although molecular features are strong, in general, in the cool and dense atmospheres of low-mass main-sequence stars, only weak $\mathrm{MgH}$ features are found in the new spectrum of SDSS J0018-0939. This confirms the low abundance of $\mathrm{Mg}$ of this object.

- New upper limits: We obtained new and stronger upper limits for $\mathrm{Zn}, \mathrm{Cu}$, and $\mathrm{Ba}$, which provide even tighter constraints on the origin of the peculiar abundances of this star.

The abundance pattern derived by the preliminary analysis for the new spectrum confirms the results obtained by our previous study. The low Si abundance suggests that the abundance pattern of this star is not well-explained by pair-instability supernovae, expected to be explosions of very massive stars $\left(140-300 \mathrm{M}_{\odot}\right)$, and core-collapse supernovae of more massive objects (Ohkubo et al. 2006) might be preferable for the progenitor (Fig. 2). The upper limit of Zn abundance would be another constraint on the progenitor. 


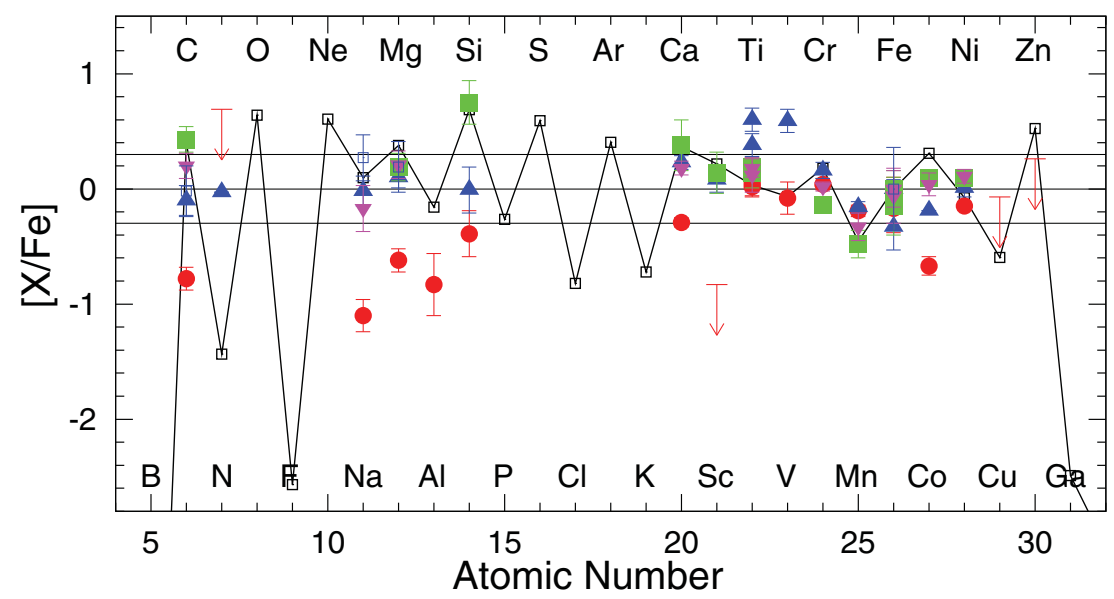

Figure 1. Chemical abundance pattern of the four cool main-sequence stars found with SDSS and Subaru observations (Aoki et al. 2010; 2013; 2014), and a comparison star G 39-36 (blue triangles: Aoki et al. 2014). The result for SDSS J0018-0939 (red filled circles) is updated by the preliminary analysis for the new spectra reported in the present work. The solid line is predictions of a core-collapse supernova model for usual massive stars (Tominaga et al., private communication). The abundance patterns of stars other than SDSS J0018-0939 are well-explained by the model, whereas SDSS J0018-0939 exhibits clear under-abundances of C, $\mathrm{Mg}$, and Co.

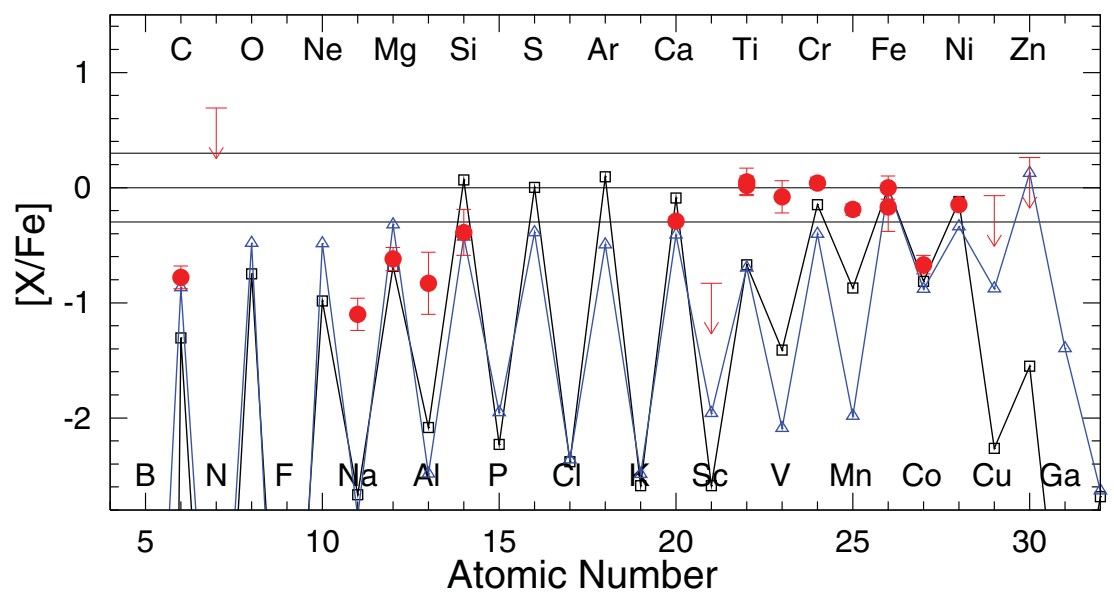

Figure 2. Chemical abundance pattern of SDSS J0018-0939 (red filled circles), compared with model predictions for a pair instability supernova of $\mathrm{a} \sim 300 \mathrm{M}_{\odot}$ star (the solid blue line with squares), and for a core collapse supernova of a more massive object $\left(\sim 1000 \mathrm{M}_{\odot}\right.$; the solid line with triangles). See text for more details.

\section{An $r$-process-element enhanced star}

Another interesting object among the four cool main-sequence stars found with SDSS/ Subaru is SDSS J2357-0052, as it exhibits large excesses of heavy neutron-capture elements (Aoki et al. 2010). Eu II lines are clearly detected in this object, and abundance ratios of neutron-capture elements (e.g., [Ba/Eu], [La/Eu]) indicate that their origin is the $r$-process. The star is classified as an " $r$-II", which have $\mathrm{Eu}([\mathrm{Eu} / \mathrm{Fe}]>+1.0)$. This is the first (and so far, unique) example of a cool main-sequence star among the $r$-II stars known to date. 
The metallicity of this star $([\mathrm{Fe} / \mathrm{H}]=-3.4)$ is the lowest among the $r$-II stars. As a result, the excess of $r$-process elements with respect to $\mathrm{Fe}$ is the highest yet found. This means that $r$-process nucleosynthesis has occurred even at such low metallicity, and the products have been preserved in some low-mass objects. Exploring the astrophysical sites of the $r$-process is currently a hot topic in nuclear astrophysics, and the distribution of metallicity and abundance ratios of heavy elements in metal-poor stars are regarded as useful constraints on the models (e.g., Wehmeyer et al. 2015; Ishimaru et al. 2015). SDSS J2357-0052 could provide one of the strongest constraints.

\section{Summary and future prospects}

Cool main-sequence stars appear to be common among very/extremely metal-poor stars, although the number of objects that have been well-studied to date is small, due to their intrinsic faintness. The sample size should increase with the advent of new surveys of very metal-poor stars in the near future, e.g., LAMOST. The fraction of cool mainsequence stars among very metal-poor stars will help determine the initial mass function for $M<0.8 \mathrm{M}_{\odot}$ stars at very low metallicity.

Our abundance analysis of four cool main-sequence stars with $[\mathrm{Fe} / \mathrm{H}]<-2.5$ discovered with SDSS confirms that typical abundance ratios derived for main-sequence turn-off stars and red giants with very low metallicity are also found in most cases. Large excesses of heavy neutron-capture elements are found for one of the four stars, and the abundance pattern is also similar to that obtained for previously reported $r$-II stars. Another star, however, exhibits very peculiar abundance patterns for the $\alpha$ and Fe-peak elements that is not well-explained by the usual core-collapse supernovae of massive stars.

There is no clear reason why stars with such remarkable abundance ratios are contained in the small sample of cool main-sequence stars. Future studies for larger samples of such objects will enable us to discuss the statistics of these stars.

Acknowledgments. This work is based on data collected at the Subaru Telescope, which is operated by the National Astronomical Observatory of Japan. We would like to thank N. Tominaga for providing us with plots shown in Figure 1 with his model calculations. W.A. and T.S. are supported by the JSPS Grant-in-Aid for Scientific Research (S:23224004). S. H. is supported by the JSPS Grant-in-Aid for Scientific Research (C:26400231). T.C.B. acknowledges partial support from grant PHY 08-22648: Physics Frontiers Center/Joint Institute for Nuclear Astrophysics (JINA), and PHY 14-30152; Physics Frontier Center/JINA Center for the Evolution of the Elements (JINA-CEE), awarded by the U.S. National Science Foundation. Y.S.L. acknowledges support provided by the National Research Foundation of Korea to the Center for Galaxy Evolution Research (No. 20100027910) and the Basic Science Research Program through the National Research Foundation of Korea (NRF) funded by the Ministry of Science, ICT \& Future Planning (NRF-015R1C1A1A02036658).

\section{References}

Aoki, W., Beers, T. C., Honda, S., \& Carollo, D. 2010, ApJL, 723, L201

Aoki, W., Beers, T. C., Lee, Y. S., et al. 2013, AJ, 145, 13

Aoki, W., Tominaga, N., Beers, T. C., Honda, S., \& Lee, Y. S. 2014, Science, 345, 912

Frebel, A., \& Norris, J. E. 2015, ARAA, in press, arXiv:1501.06921

Ishimaru, Y., Wanajo, S., \& Prantzos, N. 2015, ApJL, 804, L35

Kim, Y.-C., Demarque, P., Yi, S. K., \& Alexander, D. R. 2002, ApJS, 143, 499 
Lee, Y. S., Beers, T. C., Sivarani, T., et al. 2008, AJ, 136, 2022

Ohkubo, T., Umeda, H., Maeda, K., et al. 2006, ApJ, 645, 1352

Suda, T., Katsuta, Y., Yamada, S., et al. 2008, PASJ, 60, 1159

Wehmeyer, B., Pignatari, M., \& Thielemann, F.-K. 2015, MNRAS, 452, 1970

Yong, D., \& Lambert, D. L. 2003a, PASP, 115, 796

Yong, D., Lambert, D. L., \& Ivans, I. I. 2003b, ApJ, 599, 1357

York, D. G., Adelman, J., Anderson, J. E., et al. 2000, AJ, 1201579 